To be published in IEEE ICME 2005, Amsterdam, The Netherlands, July 2005. (c) Copyright reserved.

\title{
MEDIA AWARE ROUTING IN LARGE SCALE NETWORKS WITH OVERLAY
}

\author{
Dan Jurca, Sanja Petrovic and Pascal Frossard
}

\author{
Ecole Polytechnique Federale de Lausanne (EPFL) \\ Signal Processing Institute \\ CH-1015 Lausanne, Switzerland
}

\begin{abstract}
This paper presents a new routing strategy, that selects the best network paths in an overlay network, in order to minimize the distortion perceived by the end user. We first propose a model that reports the video distortion as a function of the encoding rate, and the loss process parameters (the packet loss ratio, and the average burst length of errors). We then derive a method to compute an accurate estimation of the end-to-end characteristics of a given path in the network topology, as experienced by the media stream. It allows for reducing a set of streaming paths between the server and the client, to a simple virtual link with equivalent end-to-end parameters. Finally, an algorithm is proposed, that finds the best streaming paths in the overlay network, in terms of media distortion. Our algorithm therefore takes into account not only the conventional network parameters (e.g. end-to-end available bandwidth), but also other application specific metrics (e.g. video distortion measure and loss process). Interestingly, it is shown that the best route in terms of video distortion, does not necessarily use the highest bandwidth links, but carefully trades off channel reliability and bandwidth.
\end{abstract}

\section{INTRODUCTION}

The Internet becomes day by day more appealing to media streaming applications, due to its continuous expansion and to the fast deployment of broadband last-mile connections to the end-users. The redundancy of the physical links in the internet, and the low utilization of their capacity potential, motivates the research of new routing techniques for media applications, that employ multiple available paths between a streaming server and a potential client.

In the same time, different criteria in choosing the paths in the streaming process, could improve the end-user experience. It has already been shown that using different metrics for routing multimedia and data traffic can lead to significant system improvements in wireless scenarios [6].

In the context of large scale networks with overlay, where some of the intermediate nodes can participate to efficient routing strategies, we propose a new routing application for multimedia traffic. Our application takes into account media aware metrics in determining the best paths used by the

\footnotetext{
This work has been supported by the Swiss National Science Foundation.
}

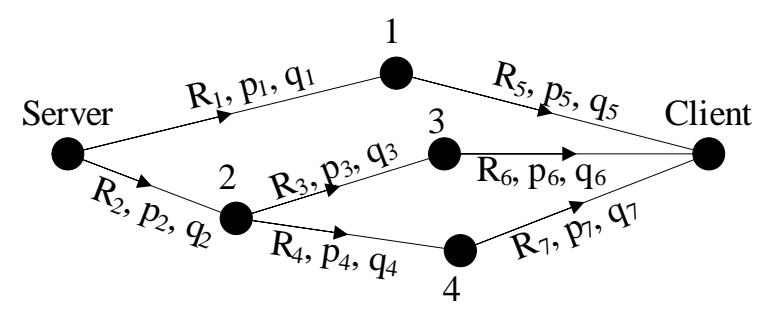

Fig. 1. Large Scale Network with Overlay.

streaming process. We use the redundancy of internet links to fulfill the bandwidth requirements of the media application [2], and we take into account other link parameters, e.g. error processes, when determining the optimal set of paths allocated for streaming [7]. Related work presents efficient methods in selecting multiple forwarding network paths for the case of multiple description video streaming $[1,4]$.

The contributions presented in this paper are threefold: first, we propose a new, general video distortion model based on the encoding source rate of the video and the error process of the intermediate transport medium (in terms of loss probability $P L R$, and average burst length $A B L$ ). Then, we propose a framework for network decomposition and simplification, whose aim is to compute a virtual endto-end model of the intermediate network with equivalent parameters in terms of rate and error process [5]. The model is later used by our routing strategy which aims at discovering the optimal set of paths to be used by a media streaming application in order to minimize the end-user distortion.

Section 2 presents our assumptions on the network, formulates the optimization problem and validates the proposed distortion model. The framework for network decomposition and simplification is presented and validated in Section 3, while our routing strategy is explained in Section 4. Section 5 concludes this paper.

\section{MEDIA AWARE ROUTING}

\subsection{System Overview}

In our large-scale network scenario we consider a media flow that is forwarded through multiple joint and disjoint network links to a client. A part of the intermediate routers can perform low level operations on the flow. e.g. splitting the incoming flow on one link, into more outgoing flows that are forwarded on different paths. This set of "active" 


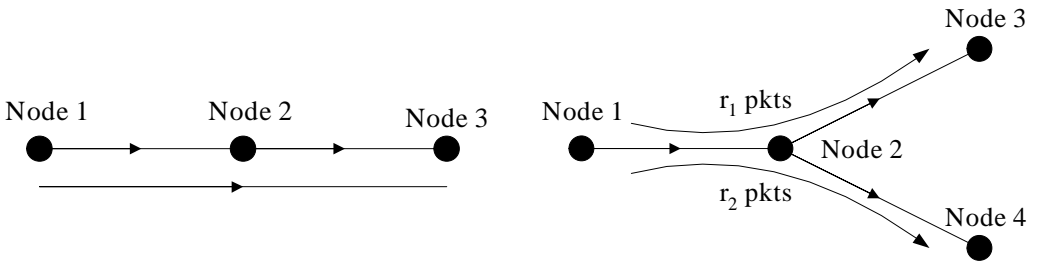

(a) Sequential Model

(b) Y Model

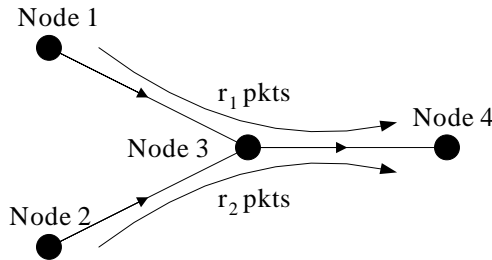

(c) Inverted Y Model

Fig. 2. Network Decomposition Building Blocks.

nodes, together with the virtual links that connect the server and the client, form an overlay network that can be represented as a directed graph $G(V, E)$ (Figure 1). The virtual links are characterized by an available bandwidth $R_{i}$ and an error process approximated as a 2-state Gilbert model with transition probabilities $p_{i}$ and $q_{i}$ (from 'good' state to 'bad' state, and from 'bad' state to 'good' state, respectively). We moreover consider the error processes between any two links from $G$, as independent.

The streamed video sequence is encoded at the required rate by a video encoder, and the resulting bitstream is packetized into network packets such that, the data relating to one frame is contained into one network packet. The flow of packets is then forwarded on the network in a FIFO order. Flow splitting across multiple paths is performed according to the available rates on each of the paths. The client decoder decodes the video frames from the received packets. In case of packet losses, the decoder will display the last successfully decoded frame.

\subsection{Optimization Problem}

In order to assess the impact of the network conditions on the streaming process, we propose a video distortion model that takes into account the network parameters as observed by the media application:

$$
D=f\left(R_{e}, P L R_{e}, A B L_{e}\right),
$$

where $R_{e}$ represents the available aggregated end-to-end bandwidth of the network graph $G$, and $P L R_{e}$ and $A B L_{e}$ represent the end-to-end packet loss ratio, and average burst length induced by the network links contained in $G$. These parameters depend on the network status and on the paths chosen by the streaming application, in a given network topology. We assume here, in a first approximation, that the end-to-end loss process can also be modelled by a single Gilbert model. The parameters of the end-to-end model clearly depend on the chosen paths in the network graph.

Therefore, given a video sequence, the distortion metric $f$, and the overlay network topology created from all available links between the server and the client, the optimization problem translates into finding the optimal subset of intermediate links, $G^{*}$, to be used by the media application, such that, the video distortion at the client is minimized:

$$
G^{*}=\arg \min _{G}(D(G))
$$

The next sections present the distortion model, and the network parameter estimation method, that will be used to solve the optimization problem.

\subsection{Video Distortion Model}

We find an expression for the distortion model based on video experiments. We encode the sequence foreman_cif (300 frames at $30 \mathrm{fps}$ ), at rates of 128, 256, 384, 512 and $640 \mathrm{kbps}$, using the H.264 codec software. We simulate the end-to-end network as a random Gilbert error process with $P L R_{e}$ between 0 and 3\%, and $A B L_{e}$ between 1 and 3 . For each of the encoding video rates, we simulate the streaming application, by averaging the results over 3000 realizations of the network, and we compare the results with the theoretical values computed according to Eq. (2):

$$
D=\xi \cdot R_{e}^{\gamma}+P L R_{e}\left(\alpha+\beta \cdot A B L_{e}^{2}\right)
$$

Parameters $\xi, \gamma, \alpha$ and $\beta$ depend on the particular video sequence and can be computed individually.

Some results are presented in Figure 3 and Figure 4. The parameters computed for the distortion model are: $\xi=$ $4.3214 \cdot 10^{6}, \gamma=-0.8876, \alpha=1624$ and $\beta=181$. The theoretical surface, closely follows the experimental points. We observe that the distortion due to packet losses increases linearly with the loss probability of the underlying network, and quadratic with the average error burst length. This fact is also confirmed by other distortion models for burst error processes [3].

\section{NETWORK DECOMPOSITION AND SIMPLIFICATION}

In order to solve the hereabove optimization problem we must now be able to compute the equivalent end-to-end parameters $\left(R_{e}, p_{e}\right.$ and $\left.q_{e}\right)$, for any network topology $G$. We create a simplified network model containing a single link with equivalent parameters, that can replace the overlay network topology between the server and the client. We attempt to approximate all network links contained in the network graph $G$, by a single link with equivalent rate $R_{e}$ and equivalent Gilbert error process $\left(p_{e}\right.$ and $\left.q_{e}\right)$. The simplified network model renders the computation of the end-user distortion tractable, while keeping an accurate image of the end-to-end network behavior.

We start form the premises that every network topology can be decomposed as a sequence of primary building blocks as represented in Figure 2. Hence, finding equivalent one-link models for each of the 3 network blocks, and 


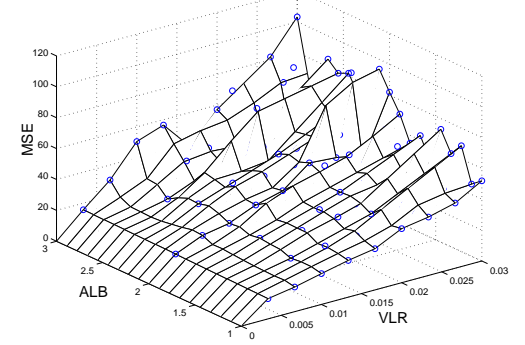

Fig. 3. Experimental Video Distortion Values.

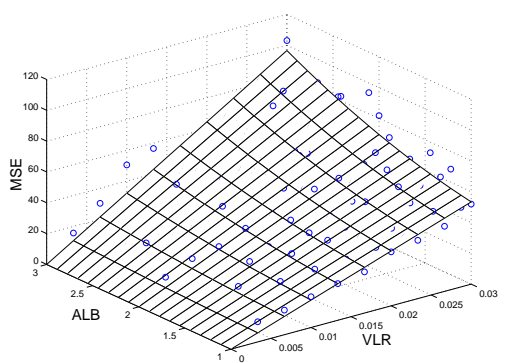

Fig. 4. Theoretical vs. Experimental Video Distortion Values.
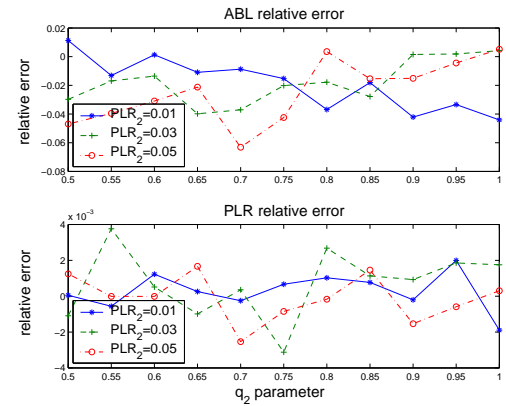

Fig. 5. End-to-end Network Model Validation vs. Experimental Results. applying the simplification iteratively, leads to the approximation of any network topology by a single link with equivalent parameters in terms of end-to-end effect.

A data flow passing through a network block as represented in Figure 2(a) is affected by the error process of both links, and is rate-limited by the smallest rate of the two links. Hence, we compute an equivalent end-to-end rate and error process for the flow [5]: $R_{e}=\min \left(R_{1}, R_{2}\right)$; $p_{e}=1-\prod_{i=1}^{2}\left(1-p_{i}\right) ; q_{e}=\frac{\prod_{i=1}^{2} P_{0 i}}{1-\prod_{i=1}^{2} P_{0 i}} \cdot p_{e} ;$ where, $P_{0 i}$ represents the steady state probability of the state "good" of link $i$, and can be computed from parameters $p_{i}$ and $q_{i}$.

A data flow passing through the network block in Figure 2(b) is split at the intermediate node 2 according to a split policy ( $r_{1}$ packets towards node $3, r_{2}$ packets towards node 4 ), into two flows going on different paths. While the rate and error process of the first link affect all packets, the rate and error process of each of the two separate links affect only the packets sent on the particular link. We compute the equivalent parameters for each of the two sub-flows for the common link, and by a similar logic as in the first case, we compute the overall parameters for each of the flows for the Y model.

Exactly computing the equivalent error parameters for each of the sub-flows will lead to a closed-form expression for $P L R_{e}$, and to a recursive expression for $A B L_{e}$ [5]. Hence, we provide an approximation for $A B L_{e}$ that accurately models its behavior. The equivalent rate and error parameters for the first sub-flow on the common link become: $R_{e}=\min \left(R_{1} \cdot \frac{r_{1}}{r_{1}+r_{2}}, R_{2}\right), p_{e}=\frac{1-P_{01}}{P_{01}} \cdot q_{e}$,

$q_{e}=q_{1} \cdot \frac{r_{1}-1}{r_{1}}+\frac{1}{r_{1}} \cdot \frac{q_{1}}{q_{1}+p_{1}} \cdot\left(1-(1-q-p)^{r_{2}+1}\right)$.

This last approximation captures the state transition (from "bad" to "good") effect on the sub-flow. The two terms in the sum relate to the two cases when a transition can occur (during the transmission of the $r_{1}$ packets of the sub-flow, or during the $r_{2}$ packets of the other sub-flow), weighted according to their probability of occurrence.

A similar reasoning is applied for the network block in Figure 2(c), in which two sub-flows aggregate into a single flow passing through a common link. The equivalent para- meters of the aggregated flow when it enters the common link are: $R_{e}=\min \left(R_{1}+R_{2}, R_{3}\right)$,

$$
\begin{aligned}
p_{e}= & \frac{q_{e}}{\frac{r_{1} \cdot q_{1}}{\left(r_{1}+r_{2}\right)\left(p_{1}+q_{1}\right)}+\frac{r_{2} \cdot q_{2}}{\left(r_{1}+r_{2}\right)\left(p_{2}+q_{2}\right)}} . \\
& \cdot\left(1-\frac{r_{1} \cdot q_{1}}{\left(r_{1}+r_{2}\right)\left(p_{1}+q_{1}\right)}+\frac{r_{2} \cdot q_{2}}{\left(r_{1}+r_{2}\right)\left(p_{2}+q_{2}\right)}\right), \\
q_{e}= & q_{1} \cdot \frac{r_{1}-1}{r_{1}+r_{2}}+q_{2} \cdot \frac{r_{2}-1}{r_{1}+r_{2}}+ \\
& +\frac{1}{r_{1}+r_{2}} \cdot\left(\frac{q_{1}}{p_{1}+q_{1}}+\frac{q_{2}}{p_{2}+q_{2}}\right) .
\end{aligned}
$$

We validate the end-to-end approximation model presented above, by simulating each of the three network blocks. We compare the theoretical results obtained by our approximations, with the simulation results obtained by averaging over 100 flows of 1000 packets for each model. We repeat the simulations for various Gilbert parameters on the links, in order to assess the cases when our approximation model fails to accurately represent the original network.

Figure 5 presents the obtained results, for the Y model with Gilbert parameters fixed on link 1 and 3 to $p_{1}=0.01$, $q_{1}=0.9, p_{3}=0.016, q_{3}=0.8$ (equivalent to $P L R_{1}=$ $\left.1.1 \%, A B L_{1}=1.1, P L R_{2}=2 \%, A B L_{2}=1.25\right)$, while varying the error parameters on the second link. We observe that the theoretical end-to-end model follows closely the modelled network in terms of $P L R$ (relative error of less that $0.5 \%$ for all simulated cases), while the relative errors in terms of $A B L$ reach a few percentage points.

We conclude that the end-to-end approximation models accurately the underlying network for reasonable scenarios of link errors of up to $5 \%$ and burst lengths of up to 1.7 . The model fails to be precise in terms of $A B L$, in the cases when a common link with very bad error parameters is split into two links with low error parameters, or when the two divergent links have error parameters different by more than $10 \%$ in absolute values.

\section{MEDIA ROUTING ALGORITHM}

Once we have the means to compute an equivalent endto-end model for any given network topology between the server and the client, we can employ a simple routing algorithm (Algorithm 1 ) that starts from the available net- 
Table 1. Parameter Values for the Links in $G(V, E)$

\begin{tabular}{|c|c|c|c|c|c|c|c|}
\hline & L 1 & L 2 & L 3 & L 4 & L 5 & L 6 & L 7 \\
\hline ABL & 1.25 & 1.25 & 1.43 & 1.11 & 1.25 & 1.43 & 1.25 \\
\hline PLR (\%) & 2.44 & 0.62 & 4.11 & 1.10 & 1.23 & 4.11 & 1.23 \\
\hline Rate $(k b p s)$ & 256 & 384 & 256 & 128 & 256 & 256 & 128 \\
\hline
\end{tabular}

work graph $G$, and parses through all possible subgraphs $G^{\prime}$, computing their end-to-end models and their associated $D\left(G^{\prime}\right)$. In the end, it will pick the routing strategy that minimizes the video distortion.

To each link $l_{i}$ in graph $G$, we attach a boolean parameter $\Phi\left(l_{i}\right)$ which indicates if the link is used or not by the streaming application. Consequently, for each link we construct a list $A\left(l_{i}\right)$ containing all subordinate links that cannot be used by the application in case link $l_{i}$ is not used. ${ }^{1}$

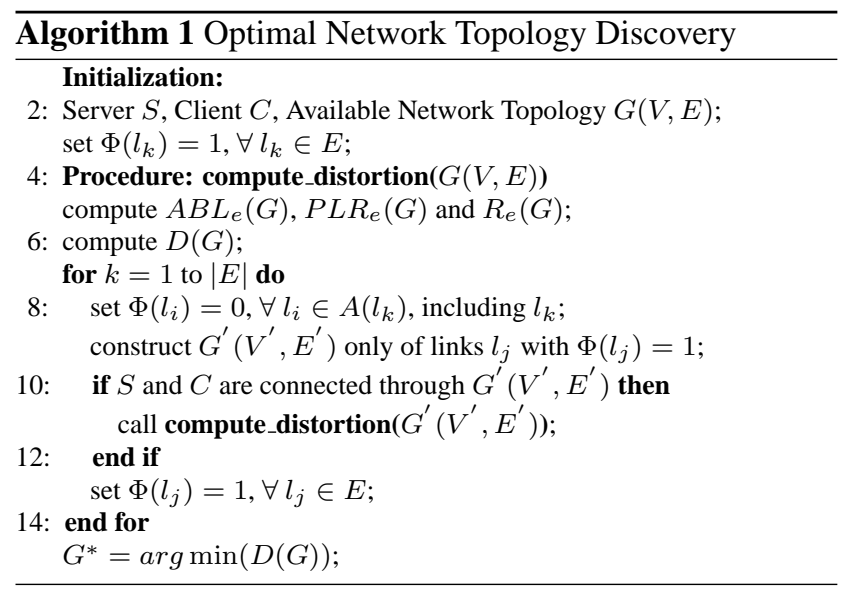

Algorithm 1 is based on pruning one by one intermediate network links, and computing resulting subgraphs. It will parse all possible network subgraphs, starting from the initial available network scenario, hence, it's complexity is combinatorial. However, in practice, the number of iterations run by the algorithm is drastically limited by the small number of network interfaces each overlay node can have, and by the small average number of hops between a server and a client in the Internet. Further complexity reductions can be made by employing smart pruning decisions (based on $A\left(l_{i}\right)$ ), so that the algorithm avoids repeating already parsed scenarios.

We test our routing algorithm on the network scenario presented in Figure 1. The parameters for all the links are presented in Table 1. We run the algorithm on all possible network graphs derived from the initial network topology, using both the theoretical end-to-end model, and the averaged simulations on the real topologies. The obtained rate and error parameters are plugged into Eq. (2). The results are presented in Table 2 .

We observe that the algorithm prefers a multipath routing solution with lower available rate (S-1-2-4-C), but better error parameters. This motivates our choice of a routing sys-

\footnotetext{
${ }^{1}$ In our network example from Figure $1, l_{5} \in A\left(l_{1}\right)$ and $l_{1} \in A\left(l_{5}\right)$.
}

Table 2. End-to-end Results For All Possible Scenarios

\begin{tabular}{|c|c|c|c|c|}
\hline Scenario & $R_{e}$ & $A B L(\exp /$ th) & $P L R(\%)$ & $D$ (MSE) \\
\hline S-2-3-4-C & 384 & $1.21 / 1.17$ & $6.78 / 6.73$ & $173.28 / 172.03$ \\
\hline S-1-C & 256 & $1.26 / 1.26$ & $3.58 / 3.65$ & $135.82 / 137.28$ \\
\hline S-2-3-C & 256 & $1.47 / 1.44$ & $8.68 / 8.63$ & $240.98 / 240.30$ \\
\hline S-2-4-C & 128 & $1.21 / 1.21$ & $2.89 / 2.93$ & $180.47 / 181.21$ \\
\hline S-1-2-3-C & 512 & $1.04 / 1.06$ & $5.99 / 6.12$ & $144.73 / 148.06$ \\
\hline S-1-2-4-C & 384 & $1.10 / 1.10$ & $3.31 / 3.43$ & $107.96 / 110.07$ \\
\hline S-1-2-3-4-C & 640 & $1.18 / 1.14$ & $5.48 / 5.52$ & $131.77 / 131.44$ \\
\hline
\end{tabular}

tem that takes into account non-conventional metrics related to the particular streaming application. Further restrictions can be applied towards limiting the number of paths used, in case such a resource becomes a constrained of the network. We also observe that our theoretical model follows with high accuracy the simulation results. The same conclusions were drawn after simulating the network topology with different link parameters.

\section{CONCLUSIONS}

In this paper, we presented a new routing paradigm for media streaming, which takes into consideration non conventional metrics related to the application itself. The presented algorithm allows the use of multiple network paths so that the streaming application can benefit from the aggregated bandwidth, while pondering the impact of link errors on the video distortion.

The introduced optimization problem is based on a video distortion model that takes into account the video source rate, along with the error process during the packet routing. The new framework for network decomposition and simplification renders tractability to the end-to-end computation of the impact of the streaming process on the video quality.

For future work, we will deepen our analysis in the sense of creating optimal scheduling policies in the intermediate nodes, and developing in-network FEC capabilities.

\section{REFERENCES}

[1] A. C. Begen, Y. Altunbasak, and O. Ergun. Multi-path selection for multiple description encoded video streaming. In Proceedings of the IEEE ICC, 2003.

[2] L. Golubchik, J. Lui, T. Tung, A. Chow, and W. Lee. Multi-path continuous media streaming: What are the benefits? ACM Journal of Performance Evaluation, 49(1-4):429-449, Sept 2002.

[3] Y. J. Liang, J. G. Apostolopoulos, and B. Girod. Analysis of packet loss for compressed video: Does burst-length matter? In Proceedings of IEEE ICASSP, 2003.

[4] Z. Ma, H.-R. Shao, and C. Shen. A new multi-path selection scheme for video streaming on overlay networks. In Proceedings of IEEE ICC, 2004.

[5] S. Petrovic. Analysis of packet loss processes in multipath networks. Technical report, EPFL, July 2004.

[6] M. B. Pursley, H. B. Russel, and P. E. Staples. Routing for multimedia traffic in wireless frequency-hop communication networks. IEEE Journal on Selected Areas in Communications, 17(5):784-792, May 1999.

[7] D. Rubenstein, J. Kurose, and D. Towsley. Detecting shared congestion of flows via end-to-end measurement. IEEE Transactions on Networking, Vol. 10, No. 2, 10(3):381-395, June 2002. 